\title{
Simulation as tools to improve wave heating in fusion plasmas
}

\author{
S. HEURAUX ${ }^{1} \dagger$ F. da SILVA $^{2}$ T. RIBEIRO ${ }^{3}$ \\ B. DESPRES ${ }^{4}$ M. CAMPOS PINTO ${ }^{4}$ \\ J. JACQUOT ${ }^{5}$ E. FAUDOT ${ }^{1}$ S. WENGEROWSKY ${ }^{1}$ \\ L. $\operatorname{COLAS}^{5}$ AND L. $\mathbf{L u}^{1,5}$
}

\author{
${ }^{1}$ Institut Jean Lamour, UMR 7198 CNRS-Université de Lorraine BP 70239 F-54506 \\ Vandoeuvre France \\ ${ }^{2}$ Instituto de Plasmas e Fusão Nuclear, Instituto Superior Técnico, Universidade de Lisboa, \\ 1049-001 Lisboa, Portugal \\ ${ }^{3}$ Max-Planck-Institut für Plasmaphysik, 85748 Garching, Germany \\ ${ }^{4}$ Laboratory Jacques Louis Lions, University Pierre et Marie Curie, BP 187, \\ 75252 Paris Cedex 05, France \\ ${ }^{5}$ CEA, IRFM, F-13108 St-Paul-Lez-Durance, France
}

(Received ?; revised ?; accepted ?. - To be entered by editorial office)

Firstly, a brief overview will be given on different models that are able to describe the behaviour of wave propagation as a function of specific frequency ranges. Each range corresponds to different heating systems, namely, 20-100 MHz for the Ion Cyclotron Resonant Heating (ICRH), 2-20 GHz for Lower Hybrid Heating or Current Drive (LHCD), and 100-250 GHz for Electron Cyclotron Resonant Heating (ERCH) or Current Drive (ECCD) systems. Every systems' specifications will be explained in detail, including the typical set of equations and the assumptions needed to describe the properties of these heating or current drive systems, as well as their specific domains of validity. In these descriptions, special attention will be paid to the boundary conditions. A review about specific physical problems associated with the wave heating systems will also be provided. Such review will detail the role of simulation in answering questions coming from experiments on magnetized plasma devices devoted to Fusion. A few examples which will be covered are the impact of edge turbulence on wave propagation and its consequences on the heating system performances, effects of the fast particles, ponderomotive effects, among others. A study that is more focused on Radio Frequency (RF) sheath effects will also be discussed. It shows that such simulations require very sophisticated tools to gain a partial understanding of the observations undertaken in dedicated experiments. To conclude this review, an overview will be given about the requirements and progress necessary to obtain relevant predictive simulation tools able to describe the wave heating systems used in fusion devices.

PACS codes: 52.35.-g, 52.50.-b, 52.55.-s,52.65.-y

$\dagger$ Email address for correspondence: stephane.heuraux@univ-lorraine.fr 


\section{General considerations on wave propagation for plasma wave-heating and current-drive}

It is useful to start citing some reviews and books that provide the background on the different kinds of waves used to heat fusion plasmas. General information about waves in plasmas can be found in Swanson's book (Swanson 2003) and Stix's book (Stix 1992); and the required Physics background on waves for heating plasmas and the early history of wave heating are available in (Cairns 1991). Reviews of electron cyclotron waves can be found in (Bornatici et al. 1983), of lower-hybrid wave in (Bonoli 1985), and of ion cyclotron wave in (Jaeger et al. 1993; Fuchs et al. 1995; Wilson \& Bonoli 2015). Having in mind the main elements of wave propagation and damping mechanisms, as well as the requirements for the ITER heating (Poli et al. 2014), the method for modelling heating systems and the last physical phenomena to take in account following new experimental evidences will be presented.

In order to model or simulate plasma heating in fusion devices, the first question to be addressed is, What are the minimal physical processes that should be taken into account? In fact, this question not at all simple, since, as the plasma is heated, its parameters change. Therefore, a self-consistent description becomes complex, due to the fact that transport is, at least, a function of the local temperature gradients that are modified by the wave energy deposition, which is itself a function of the plasma parameters' local values. In previous work, we have only considered smooth plasma parameters, which used to be the standard approach. Recently, the role of density fluctuations started to be seen as an important parameter, especially at the plasma edge, in a passive way, as well as for the electron cyclotron heating (Decker et al. 2012; Tsironis et al. 2009) or the lower hybrid current drive (Peysson \& Decker 2014). It also affects diagnosis that use waves, since it changes the properties of the probing beam at an higher order. At the next order, wave trapping effects, multi-scattering and non-linear reflection are introduced (Heuraux et al. 2010; da Silva et al. 2010). Possibilities to generate parametric instabilities during heating (Gusakov et al. 2014) or mode conversion to remove accessibility conditions associated with cut-offs (Irzak \& Popov 2008) are reconsidered. In these last cases, the code should describe multi-modes propagation, and the plasma domain can be reduced to a poloidal cross-section, due to very small parallel wavenumber values for the turbulence. Considering these remarks is enough to: (i) deduce that the minimal set of equations driving the wave propagation should be written at least in $2 \mathrm{D}$; (ii) be able to integrate fluctuating plasma parameters; and (iii) describe several modes simultaneously. That is to say a relevant code should compute all electric field components in $2 \mathrm{D}$ for a given parallel wavenumber. The radiofrequency sheath effects have to be integrated in the simulation of Ion Cyclotron Resonant Heating simulations through the particular boundary conditions to describe the spurious effects seen in experiments Wukitch et al. (2009); Jacquet et al. (2011) that requires to compute slow and fast wave fields at the same time. In general, the available codes offer a single-mode or multi-mode description with some sophistications, such as a non-Maxwellian distribution function (Dumont \& Zarzoso 2013; Brambilla \& Bilato 2009; Peysson \& Decker 2014) and ad-hoc tools to cross a wave resonance. Concerning this last point, new mathematical developments allow for solutions without the introduction of artificial dissipation (Després et al. 2014). 


\section{Wave-heating simulations for fusion plasmas}

\subsection{Introduction}

An initial idea on the approximations done to describe wave propagation using Maxwell's equations can be the understanding of the idealized model analysis written in Jackson's book (Jackson 1999). With that, it is possible to create a clear picture of the different approximation levels. At the same time, a second question arises, which is: How to describe the behaviour of the medium versus the studied wave? A third question is: How to do it without missing a relevant mechanism? To address them, more and more Physics needs to be taken into account. This increase of the physics contain induces an increase of the computation time, which can go above the possibilities of the actual computer capabilities. So a compromise has to be found to work at same computation time between the physical inputs and numerical accuracy, that is to say the number of grid points or finite elements. That has an impact of the exactness of the simulation done. This point induces questions: How to achieve the best numerical description of a given heating experiment whilst meeting the numerical constraints (computation time, stability, accuracy)? How to know if there are missing constraints (for example voltage limitation to avoid arcing) or not? How to introduce the technical constraints when one wants to optimize a heating system or attaining a given objective as the induced plasma current in a given ITER scenario? An example to illustrate this is to find an optimal efficiency as a function of the incident angle of the mirror, knowing the energy deposition into the plasma and the depolarization rate, which depends on the material aging of the mirror that stays unknown if even if to prevent this problem a cleaning mirror system is designed, at this moment, under the fusion plasma exposure. In fact, we are far from the possibility of optimizing a heating system due to the lack of information, for example, the ITER plasma density profile in front of the ICRH antenna is not available, knowing that this input is essential to determine the properties of the plasma-antenna coupling. Another distant possibility is to find the set of parameters in which a given heating system is able to work, following the specifications of an experiment. It is still not possible to determine the margins in which this experiment can be performed, knowing that the heating induces changes to the plasma parameters, including the turbulent transport and the magneto-hydrodynamic (MHD) activities. As a partial conclusion, the description of the wave propagation, including absorption (damping), requires the use of Maxwell's equations and adapted partial differential equations that describe the response of the plasma. These equations should be linear or non-linear, depending on the wanted requirements and the working conditions. In any case, the geometry of the system should be preserved, in order to keep the exact boundary conditions regarding the possibility of having oblique RF sheaths. These RF sheaths strongly change the behaviour of the slow wave in front of an Ion Cyclotron Antenna. The wave polarisation's role on the damping mechanisms is essential. Therefore, the next generation of codes describing a wave heating system needs to include a multi-mode description able to follow the wave polarization changes in order to optimize the energy deposition into the plasma. Premise that future development the role of the turbulence on the linear mode conversion efficiency in O-XB heating scheme was recently published (Popov 2015) or further developments of the SWITCH code for ICRH heating (Jacquot et al. 2014). Other points have also to be considered in the time-dependent cases, where Maxwell's equation solvers are coupled with J-solvers, the numerical stability and the energy conservation. The both points become problems in the large number of iterations, although they are still below what is required for ITER (da Silva et al. 2015). This kind of numerical problems disappears when the time-dependent part is removed under the following assumptions: the launch wave is con- 
sidered as monochromatic (continuous emission at the same frequency) and the plasma parameters present time-scale evolution much larger than the time of flight of the heating wave. Extra requirements should be considered for the solvers which should be able to describe wave propagation in inhomogeneities present in experimental configurations as well as high level of plasma fluctuations and to be able to cross wave resonances. The previous assumptions are generally used in the cases of wave heating simulations, which require also to describe source terms and boundary conditions in a relevant way. To finish to provide relevant information about the behaviour of the heating system and its impact on the plasma parameters, these solvers have to be included in an integrated tokamak modelling framework, but they have to be optimized before their integration into such platform.

\subsection{Basic equations}

In the most general situation, Maxwell's equations are used and can be written as follows:

$$
\begin{aligned}
\nabla \cdot \overrightarrow{\mathbf{E}}=\frac{\rho(\overrightarrow{\mathbf{E}}, \overrightarrow{\mathbf{B}})}{\epsilon_{0}} & \text { Poisson's law } \\
\nabla \cdot \overrightarrow{\mathbf{B}}=0 & \text { magnetic flux conservation law } \\
\nabla \times \overrightarrow{\mathbf{E}}=-\frac{\partial \overrightarrow{\mathbf{B}}}{\partial t} & \text { Faraday's law } \\
\nabla \times \overrightarrow{\mathbf{B}}=\mu_{o} \overrightarrow{\mathbf{J}}(\overrightarrow{\mathbf{E}}, \overrightarrow{\mathbf{B}})+\frac{1}{c^{2}} \frac{\partial \overrightarrow{\mathbf{E}}}{\partial t} & \text { Ampère's law }
\end{aligned}
$$

plus PDEs describing the density current $\overrightarrow{\mathbf{J}}$ and charge density $\rho$ evolutions as function of $(\overrightarrow{\mathbf{E}}, \overrightarrow{\mathbf{B}})$ where $\epsilon_{0}$ is the dielectric constant of vacuum, $\mu_{o}$ is the permeability of vacuum and $c$ is the speed of light in vacuum. Here, we consider only the plasma parameter set of magnetic fusion, meaning the plasma quantum effects are neglected and first order relativistic corrections are taken into account. In this framework, the most general description of plasma responses to wave excitation is driven by Vlasov's equation. This includes kinetic effects able to describe precisely all the wave-particles interactions as Landau's damping or cyclotron resonances. Since the plasma is composed of different particle species, for each kind of particle, one Vlasov's equation should be written and coupled through Maxwell's equations or introduce a Fokker-Planck equation as described in (Eester 2012). Therefore, the set of PDE necessary to define $(\overrightarrow{\mathbf{J}}, \rho)$ can be written as follows:

$$
\frac{\partial f_{s}}{\partial t}+\overrightarrow{\mathbf{v}_{\mathbf{s}}} \cdot \nabla_{\overrightarrow{\mathbf{r}}} f_{s}+\frac{q_{s}}{m_{s}}\left[\overrightarrow{\mathbf{E}}+\overrightarrow{\mathbf{v}_{\mathbf{s}}} \times(\overrightarrow{\mathbf{B}})\right] \cdot \nabla_{\overrightarrow{\mathbf{v}}} f_{s}=0
$$

with

$$
\rho(\overrightarrow{\mathbf{r}}, t)=\sum q_{s} \int d \mathbf{v} f_{s}(\overrightarrow{\mathbf{r}}, \overrightarrow{\mathbf{v}}, t), \overrightarrow{\mathbf{J}}(\overrightarrow{\mathbf{r}}, t)=\sum q_{s} \int d \mathbf{v} \overrightarrow{\mathbf{v}} f_{s}(\overrightarrow{\mathbf{r}}, \overrightarrow{\mathbf{v}}, t),
$$

where $q_{s}$ is the charge, $m_{s}$ is the mass, $\overrightarrow{\mathbf{v}_{\mathbf{s}}}$ is the particle velocity, and $f_{s}$ is the distribution function in phase space of the species $\mathrm{s}$.

In the $3 \mathrm{D}$ case, this PDE set (2.5) is too demanding in terms of computational resources. Thus, one needs to remove part of the Physics by adding relevant assumptions, whilst preserving the main effects of wave injection for heating or current-drive, even if you can use near to far field transformation (Taflove \& Hagness 2000). That means that one has to focus on specific studies and forget, momentarily, having a universal tool able to describe the wave heating system in its entirety. To take into account all the possible 
synergy available or the spurious effects generated by the wave itself, such as the plasma density fluctuations, one needs a suite of integrated codes assumed to converge, which is not at all guaranteed. To be practical, we now focus on possible assumptions to reduce the complexity of the model, first in general, and after for each wave heating system.

The most common assumption within a good framework for the wave propagation for a polarization of the launched wave is the cold plasma approximation. Generally, it is mostly because the hot plasma corrections are small. The relativistic corrections has to be taken into account earlier than the hot plasma corrections when the electron temperature becomes greater than a few $\mathrm{keV}$ (Bindslev1991) and these relativistic effects can be introduced through an electron mass correction. The relativistic effects induces an increase of the effective mass of the electron, which drives a frequency down shift of the electron cyclotron frequency. The damping processes in tokamak plasma are essentially a result of a resonant interaction between waves and particles, which requires to know the velocity distribution function in an accurate manner. That means the cold plasma approximation is inappropriate to describe the wave absorption in the tokamak plasma core. To compute accurately the damping rate a good knowledge of the velocity distribution function which is generally provided by a Fokker-Planck equation, which permits to introduce the trapped particles contributions. A detailed discussion on this point can be found in (Gnesin 2011) for electron cyclotron heating, in (Peysson \& Decker 2014) for the lower hybrid heating and current drive, and in (Brambilla \& Bilato 2009) for the ion cyclotron resonant heating.

Usually in wave heating simulation the monochromatic wave assumption is used, in this case, the most used solver to describe wave propagation allows taking into account the interferences, the scattering, and the multi-reflection effects. This solver can describe a single wave equation for a given polarization or multi-modes depending on the computed components of the electric field. This solver type is called full-wave, and corresponds to a Helmholtz-like equation, which comes from Maxwell's equations or equivalent in terms of $\overrightarrow{\mathbf{A}}$ and $\phi$ and reduces to:

$$
\left\{\begin{array}{l}
\left.-\nabla \times \nabla \times \overrightarrow{\mathbf{E}}(\mathbf{r}, t)+\frac{\omega^{2}}{c^{2}}(\overrightarrow{\mathbf{E}}(\mathbf{r}, t))+\frac{i}{\omega \epsilon_{o}} \overrightarrow{\mathbf{J}_{\mathbf{p}}}(\overrightarrow{\mathbf{E}})\right)=-i \omega \mu_{0} \overrightarrow{\mathbf{J}}_{\text {ant }}\left(\mathbf{r}_{\text {ant }}\right) \\
\overrightarrow{\mathbf{J}_{\mathbf{p}}}(\overrightarrow{\mathbf{E}})=\overline{\bar{\sigma}} \cdot \overrightarrow{\mathbf{E}} \quad(\text { linear case }) \text { or } \overrightarrow{\mathbf{J}_{\mathbf{p}}}(\overrightarrow{\mathbf{r}}, t)=\sum q_{s} \int d \mathbf{v} \overrightarrow{\mathbf{v}} f_{s}(\overrightarrow{\mathbf{r}}, \overrightarrow{\mathbf{v}}, t),
\end{array}\right.
$$

where $\overline{\bar{\sigma}}$ is the cold or hot plasma conductivity tensor corresponding to the heating frequency $\omega . \overrightarrow{\mathbf{J}}_{\text {ant }}$ is the current density source term corresponding to the antenna emission, for which the density current expression in space should represent the effective wavenumber spectrum of the antenna, taking into account the plasma properties in front of it. It is clear that, for a hot plasma description, the evolution of the plasma conductivity is taken into account and it is generally done using or Vlasov's equation or FokkerPlanck equation as mentioned before, which can also include other heating contributions through adapted quasi-linear coefficients. Another is also explored using Particle In Cell code coupled to Maxwell's equation and use of an implicit scheme overcomes the stability problem (Smithe 2007). However the needs in terms of computational resources can become intractable in ITER cases.

\subsection{Numerical tools, source terms and boundary conditions}

A recent review describes shortly the numerical techniques and schemes developed to compute the properties of the different heating schemes (Eester 2012). A brief recall of the different kind of wave solvers can be found in (Heuraux et al. 2014; Heuraux \& da Silva F. 2012), in order to clarify what are their relevant domain in wave heating. 
However, several important points still have to be discussed in order to complete the landscape of wave heating simulations in magnetized plasmas: the source terms and the boundary conditions. Usually, the boundary conditions are specific to a kind of heating, which can be very different from the standard electromagnetic boundary conditions as Perfect Electric Conductor (PEC) or others described in Taflove's book (Taflove \& Hagness 2000). A complex boundary condition exists in the case of ICRH due to the difference between electron and ion mobility, indeed a charge separation can be induced and modifies the parallel electric field component (parallel to the DC magnetic field). That changes the behaviour of the wave against the metallic wall, and at the same time generates a rectified DC potential, due to a lack of electrons induced by the RF electric field. Therefore, it is necessary to introduce sheath boundary conditions for the ion cyclotron heating to take into account the fact that the parallel component of the incident wave can be more or less screened at the wall. This depends on the incident angle of the magnetic field line on the wall and on the local values of the plasma parameters (Kohno et al. 2012; Jacquot et al. 2014). We will further develop this concept of boundary conditions later on in Section 3.

Another boundary condition is often used to reduce the mesh size, which corresponds to free wave propagation through the mesh edge without reflection, which is the so-called Perfect Matching Layer (PML) boundary condition (Bérenger 1994). Although very useful, the PML boundary condition doesn't work in anisotropic media, with propagating modes having opposite phase velocities or negative value of components in the dielectric tensor (Jacquot et al. 2013), as it is for the meta-material. A refine computation of the near fields in a case of wave heating can be possible using PML layers assuming that the single-pass absorption is valid. The spatial domain is then reduced to the region where the near-fields are of interest (Jacquot et al. 2014). The implementation of the PML layers depends on the working domain, frequency or time domain (Taflove \& Hagness 2000).

In order to define the boundary conditions appropriately (PEC, PML, ...), an exact knowledge of the geometry of the launching structure is required. In the case of the sheath boundary condition the knowledge of the angle magnetic field line and the wall is essential that requires to have a good description of the antenna design and on the magnetic configuration. This is particularly important at the grazing incidence, when the Debye sheath disappears. As a consequence, there is no charge separation on average (Stangeby 2012) and the rectification of the RF potential is reduced or disappears as shown using 2D Particle in Cell simulations depending of the RF potential amplitude (works under way in the framework of an EURO fusion enabling research contract). The complete knowledge of the antenna structure and surroundings is also needed in order to compute the near-fields in front of it. This computation is essentially devoted to the so-called plasma-antenna coupling codes.

Then, its map can be used as an input in the wave equation solver as a source term. Generally speaking, this kind of source terms is provided by antenna-plasma coupling codes to stay as close as possible to the experiments. Depending on the studies, synthetic source terms can be implemented as a Gaussian beam or anything else one wants. However, some caution has to be taken to avoid bi-directional emission $(-\overrightarrow{\mathbf{k}}$ and $+\overrightarrow{\mathbf{k}}$ at the same time). Having unidirectional emission of the launching wave is less simple to implement or requires sophisticated tools if one wants to introduce a transparent source (da Silva et al. 2005; Taflove \& Hagness 2000). To simulate an antenna in interaction with 
its surroundings, the source term should be as close as possible to the hardware components to simulate accurately the radiation wave pattern of the wave launcher. This would allow to inject the power into the antenna, for example, to study the resonances between a horn antenna put in a cavity between two ITER blanket modules (da Silva et al. 2006a). At this moment, the wave launcher will be equipped of diagnostics to measure the changes in the density profile in front of the antenna to study the interplay between the edge plasma parameters and the injected power in the cases of IRCH and LHCD, which is not actually well included in the present wave heating simulations and the changes are required to understand the plasma-antenna coupling.

\subsection{Heating simulation}

In general, as the launched frequency is fixed for a given plasma shot, the wave description can move to the frequency domain, which is generally more comfortable than the time domain that normally has to fulfil a CFL's condition (Courant et al. 1928) to be numerically stable. A first problem on the exactness can appear after this choice, especially when two or more modes exist in the computation domain at the same time for this chosen frequency as it is for the ICRH simulations where slow and fast waves exist at the plasma edge (Colas et al. 2012; Jacquot et al. 2014). A question then arises, Are these modes decoupled or not, and if not, how are them translated in terms of mode coupling or linear mode conversion? For example, the Ordinary mode (O-mode) and Extraordinary mode (X-mode) can be coupled linearly through density parallel to the magnetic field or its fluctuations (Colas et al. 1998), and a wanted (or not) depolarisation of the launching wave can occur. This phenomenon can possibly take place during electron cyclotron heating and it can be used after a good choice of the oblique incidence of the launching wave on O-mode, as done in the O-X-B double mode conversion heating scheme (Laqua et al. 1997). In such heating scheme, two questions are still open. The role of the density fluctuations in the conversion regions (Popov 2015) and the effect of the beam widening induced by the fluctuations at the edge, as was shown for ITER electron cyclotron heating cases (Sysoeva et al. 2014; Peysson \& Decker 2014). Following this example and trying to answer these questions, we need to look at the different wave heating systems independently, since each frequency ranges are indeed separated from each other.

Now, we focus on heating in tokamaks. Three main types of solvers can be identified: ray tracing, Helmholtz' equation, and Maxwell's equations corresponding to different orders of approximations.

Just an up-to-date on the simulations using the highest level of approximation associated to a ray tracing code corresponds to a single-mode description in the WKB approximation (Swanson 2003), which includes no cut-off (local index equal to zero), no resonance (local index value going to infinity) in the computing, and only where the wave propagation is described without interferences, scattering or mode conversion. An additional equation can be added to provide the spatial evolution of the amplitude, as it is, for ray tracing code in toroidal geometry with adapted coordinate system, as it was done in (Peysson et al. 2012; Marushchenko et al. 2014) or for Gaussian beam (Bertelli et al. 2012). While the dispersion relation is known, it is possible to apply this tool if the initial conditions are also known. The vacuum-plasma border crossing should be treated very carefully. A stopping condition based on the wave absorption can be added, which permits, at the same time, to determine where the energy is deposited. This does not take into account scattering, cavity effects, mode conversion, or interference pattern. Surprisingly, the obtained results using ray tracing code provide an overall good behaviour of the wave. 
Ion Cyclotron, Lower-Hybrid, and Electron Cyclotron Heating codes

ICRH codes: a brief description of the full-wave codes used for the ion cyclotron frequency heating simulation is given in (Budny et al. 2012). In a variational formalism used in EVE code (Dumont \& Zarzoso 2013) there is no possibility to model the antennaplasma coupling self-consistently, because at this moment the density current on the antenna is fixed and cannot be modified by the current induced on the antenna structure, which is limited to the straps description. To obtain relevant near-field computations, other ways are used to build antenna-plasma coupling codes, for example, Boundary Element Method in ICANT code, and integral method in TOPICA (see (Pécoul et al. 2002; Lancellotti et al. 2006)). Another one the TORIC code, using spectral decomposition, was also developed to study the ICRH scenarios (Brambilla 1999). For the SCENIC code it is described in (Jucker et al. 2011), where the full-wave solver part should be improved to become relevant to ITER and stellarator applications. These codes follow a suite of home-made code, previously mentioned for ion cyclotron, plus the TOMCAT code and its upgrades, in which a coupling code is included (van Eester \& Koch 2001). Recently, commercial software packages are being used to solve the Helmholtz equation applying the Finite Element Method (FEM). For instance, COMSOL is used in many cases: the ion cyclotron in SSWICH code (Jacquot et al. 2013), and Lower-Hybrid cases (a-b) .

LH codes: Two codes based on COMSOL software deal with the lower-hybrid heating (a) the most recent the LHEAF code (Shiraiwa et al. 2010) and (b) the coupling code PICCOLO2D including non-linear effects (Preynas et al. 2013), which is based on the same principle as the 1D code ALOHA (Hillairet et al. 2010). The lower hybrid wave propagation code TORLH (Wright et al. 2008) follows the same numerical method as TORIC code. The coupling code TOPLHA (Milanesio et al. 2012) uses the same tools developed in TOPICA code. To study the Lower-Hybrid Current Drive a ray-tracing code coupled to Fokker-Planck solver has been used including the effect of density fluctuations, which can also be applied for studying Electron Cyclotron Current Drive (Peysson \& Decker 2014). An up to date review of the Lower-Hybrid Heating and Current Drive is found in (Bonoli 2014).

EC codes: In the frequency range of electron cyclotron heating, the Helmholtz solvers were used for diagnostics (Fanack et al. 1996; Mazzucato 1998) a long time ago. Until recently, the ray tracing method was estimated to be enough to study a beam propagation in ITER plasmas (Prater et al. 2008), but it is clear now that the density fluctuations have to be included (Decker et al. 2012), and, for these studies, full-wave codes are required for the frequency range of electron cyclotron heating or current drive (Sysoeva et al. 2014, 2015).

Before going further, a remark has to be made on the commercial software available, since the tendency to use multi-physics software is becoming stronger and stronger. This can induce problems if specific difficulties associated with new types of numerical schemes are needed that are not implemented in the commercial software, such as the asymptotic preserving scheme for very high anisotropy (Crouseilles et al. 2013), just like the dielectric tensor of a magnetized plasma for ICRH. Furthermore, new developments of no interest for industries can lead to a lack of numerical tools in commercial software. For example, PML in magnetized plasma or unsolved numerical instabilities found in it (Heuraux et al. 2014). Their use on High Performance Computer can be another source of difficulties due to a commercial policy "one licence per node".

The next degree of sophistication is to obtain direct response of current density, $\overrightarrow{\mathbf{J}}$, and 
the charge separation, $\rho$, using a set of differential equations driving their time-evolution during the heating, to insert as the source terms in Maxwell's equations. Depending on the degree of approximation, one is able to describe cold plasma linear response in the case of the equations of motion of particles (Després et al. 2014), or kinetic responses for the charge separation $\rho$ and the current density $\overrightarrow{\mathbf{J}}$ as defined for the Eq.(2.5) in the case of the Poisson-Vlasov equations, with one equation for each species (Kuley et al. 2013), or Particle In Cells (PIC) code in (Smithe 2007; Smithe et al. 2014). These last descriptions based on the mean field theory allow describing, in principle, non-linear and boundary condition effects, but they require HPC machines. There are numerical stability problems due to the anisotropy introduced by the external DC magnetic field (Heuraux et al. 2014). One is connected to the very high difference between responses parallel to the magnetic field and those perpendicular to the magnetic field, and another relates to the accumulation of round-off error on the long computation time (more than $10^{6}$ time steps) that drives numerical instabilities as shown in (da Silva et al. 2015). On such long runs, the energy conservation should be fulfilled to preserve the statistical properties of the physical system. This is especially true if you want to study, after averaging, the macroscopic effects of turbulence on the wave propagation. This last part is an on-going project showing that new methods improve the wave propagation descriptions (Tierens \& Zutter 2012) and the PIC code responses (Campos Pinto et al. 2014) or gyrokinetic responses using an asymptotic preserving scheme (Crouseilles et al. 2013).

Up to now, we had only introduced the elements needed to describe the wave propagation in a given plasma, considering, more or less, the local modifications of the plasma properties. To see the full impact of the heating on the target plasma, the equilibrium and transport equations have to be solved in parallel. This is necessary in order to provide the new values of plasma parameters to the wave heating codes, including an equation of density current transport (in the case of current-drive (Peysson \& Decker 2014)). Due to the role of density fluctuations at the edge on the wave heating performance, a turbulence code also has to be used. This can be done iteratively using an integrated tokamak modelling, as in CRONOS (Artaud et al. 2010) or in the European ITM platform (Falchetto et al. 2014). At this moment, we are rather far from this situation. The focus of the effort should be on improving the depolarisation mechanisms and the performance of each module (particularly on wave solvers), in which specific boundary conditions have to be implemented. Now, we present two points that are under development, the sheath boundary conditions in magnetized plasma, and how the role of the density fluctuations can be simulated.

\section{Sheath boundary conditions (SBC)}

\subsection{Context}

The wave propagation of radio-frequency $(\mathrm{RF})$ waves, radiated by complex antennae, and their energy deposition in the core of magnetized plasmas, have been described, for a long time, using sophisticated first principle models in realistic geometry, as mentioned in the previous paragraph. Nevertheless, these simulations do not explain the experimental facts seen at the plasma edge during ion cyclotron heating (Jacquet et al. 2011; Jacquot et al. 2014). Comparatively, the simulation of anomalous RF power losses in the plasma edge is still less advanced, although the non-linear wave-plasma interactions in the plasma edge often set the operational limits of RF heating systems. Peripheral wave damping in the ion cyclotron range of frequencies $(30-100 \mathrm{MHz}$ in present fusion devices) is attributed to a DC 
biasing of the edge plasma by RF-sheath rectification induced by the different mobility of ions and electrons (Noterdaeme \& Oost 1993). The first modelling of this non-linear process was provided by (Perkins 1989), in an analogy of a double Langmuir probe driven by an oscillating RF voltage, estimated as the field-line-integrated RF field $E_{\|}$parallel to the confinement magnetic field $B_{0}$. However, in this kind of models, each open flux tube in the scrape-off layer (SOL) is considered independent of its neighbours. The non-linear coupling between flux tubes can be found in (Faudot et al. 2006). Its effects for ICRH ITER antenna are available in (Faudot et al. 2010). The main ingredient to determine the RF potential along a magnetic field line $E_{\|}$is generally computed from conventional antenna codes in the absence of sheaths, i.e., perfect electric conductors are assumed in direct contact with the plasma. This procedure, although clearly not self-consistent, was widely implemented as the only tool able to model realistic wave-launching structures (Pécoul et al. 2002; Lancellotti et al. 2006). The theoretical background of the SSWICH code that considers sheath boundary condition is in (Colas et al. 2012). However, only magnetic field lines perpendicular to the wall are considered in that paper. A crude model of the oblique sheath boundary conditions is described in (Kohno et al. 2012). A recent work on sheaths in magnetized plasmas mentions that the Debye sheath disappears at grazing angle (Stangeby 2012). Consequently, the entire DC potential drop is associated with the magnetic presheath. To evaluate the impact of the oblique sheath boundary conditions, PIC simulation using VORPAL (commercial software) has been reviewed in Smithe et al paper (Smithe et al. 2014). This simulation, when done on a tiny domain, gives the feeling of this phenomenon and provides accurate Physics, but without the possibility of being relevant for ITER cases in the near future due to the computation requirements knowing that a $10 \times 10 \mathrm{~cm}$ area needs $10^{7}$ particles per species to have a significant result above the noise level. Recent experiments introduce new facts in contradiction with the existing models, e.g., the scaling of rectified DC plasma potential with RF power (Wukitch et al. 2009), the radial penetration of the plasma bias (Faudot et al. 2010) or the non-linear generation of edge DC currents by RF waves (Bobkov et al. 2010), for which a fluid model has been built (Faudot et al. 2013). Although no consensus presently exists over an alternative approach, RF-sheath physics needs improvements using the first principles.

\subsection{Modelling and Results}

The proposed SSWICH model is thought of as a minimal self-consistent RF+DC approach, able to capture the experimental phenomenology. It was motivated by a closer proximity to the first principles and the allowance for DC current circulation in the SOL. The model involves DC plasma quantities as well as harmonic RF waves oscillating at the IC wave frequency $\omega_{0}$. RF and DC quantities are coupled non-linearly via two processes representing the sheath physics and implemented as boundary conditions. The plasma medium is still highly anisotropic high conductivity in the parallel direction. However, the neighbouring field lines are coupled via RF and DC current exchanges and, if they are sufficiently long, their two extremities can behave independently. Being minimal, the model is not fully complete in its present simplified formulation, with a single mode description associated to the slow wave that is assumed to be the source of the RF potential $V_{R F}$ driving the rectification process, following the expression:

$$
V_{R F}=\int E_{\|} d s
$$

where $s$ is the curvilinear coordinate associated with a magnetic field line and $E_{\|}$is the parallel electric field of the slow wave. Due to the difference between ion and electron 


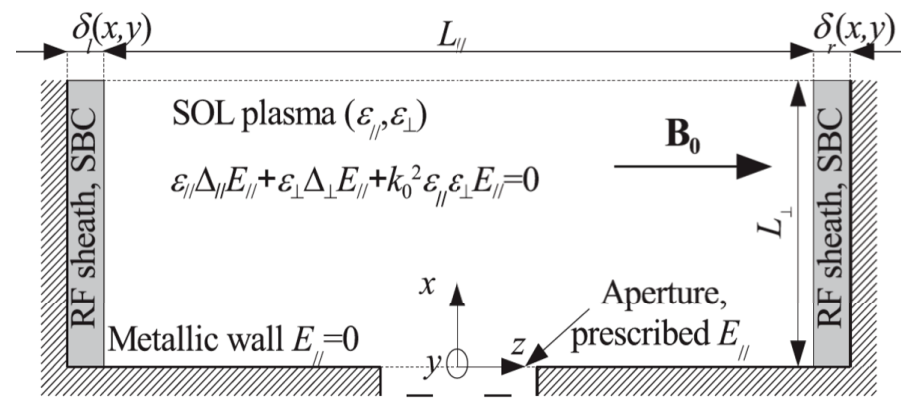

FiguRE 1. RF model to describe the emitted wave taken into RF sheath boundary conditions

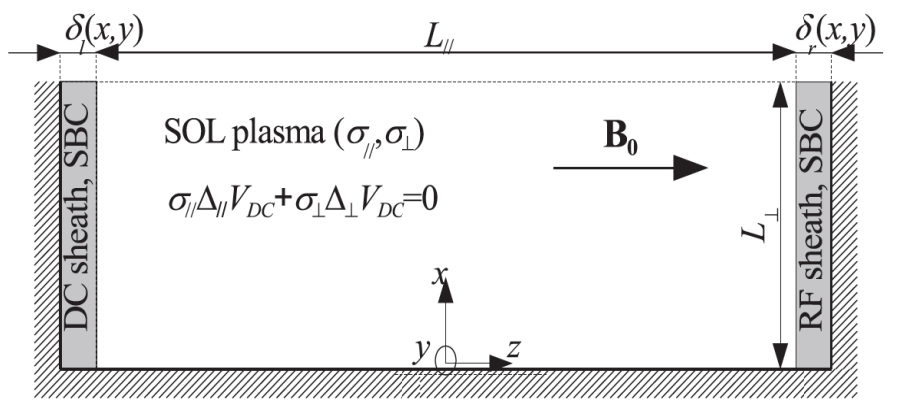

FIGURE 2. DC model part associated to RF sheath boundary conditions

mobility, a DC potential is generated, on average, over a period of the wave heating frequency. This DC potential modifies the sheath width, which changes the capacitance and the resistance of the sheath, e.g., the electric properties of the sheath against the $\mathrm{RF}$ electric field of the slow wave. With this scenario, it is clear that a self-consistent treatment is required to describe the slow wave interaction with the wall, assuming that the magnetic field lines are perpendicular to the wall. This last assumption is not always valid, and the future description with oblique incidence to the wall should be done in more complete versions than those given in (Kohno et al. 2012), where the sheath is associated to a dielectric constant corresponding to vacuum. Both figures Fig.1-2 describe a crude model of an antenna, for which, we want to introduce sheath boundary conditions. The model equation driving the slow wave is written in Fig.1, and the charge exchange in Fig.2. The RF part of the model shown in Figure 1, where $\mathrm{x}, \mathrm{y}, \mathrm{z}$ denotes, respectively, the local radial, poloidal, and parallel directions of a flattened tokamak. The simulation is a 3D collection of straight open field lines aligned along $\mathrm{z}$ in the SOL. For the sake of simplicity, Cartesian geometry will be considered here, with parallel (respectively, radial) extension $L_{\|}\left(L_{\perp}\right)$. The equations for the 3 physical quantities are coupled together by sheath boundary conditions (SBC) applied on all lateral boundaries as indicated on Fig.1, and can be written as follows:

$$
\begin{gathered}
\varepsilon_{\|} \Delta_{\|} E_{\|}+\varepsilon_{\perp} \Delta_{\perp} E_{\|}+\varepsilon_{\|} \varepsilon_{\perp}\left(\omega_{0} / c\right)^{2} E_{\|}=0 \text { [Slow Wave propagation], } \\
\varepsilon_{\perp} \Delta_{\perp} V_{R F}= \pm \varepsilon_{\|} \partial_{\|} E_{\|}[\mathrm{RF} \text { voltage excitation from RF fields] } \\
\sigma_{\| D C} \Delta_{\|} V_{D C}+\sigma_{\perp D C} \Delta_{\perp} V_{D C}=0 \text { [DC charge conservation w/o sources]. }
\end{gathered}
$$

In these equations, $\varepsilon_{\|}$and $\varepsilon_{\perp}$ are the diagonal elements of the plasma dielectric tensor at the RF pulsation $\omega_{0}, \sigma_{\| D C}$ is the DC Spitzer parallel conductivity, and $\sigma_{\perp D C}$ is a small effective DC perpendicular conductivity. The SBC expression comes from the fact, 
at the RF frequency, that the RF current through the sheath is supposed to be mainly a displacement current. In this framework, the sheath is assimilated to a parallel plate capacitor of width $\delta$, filled with a dielectric material of dielectric constant $\varepsilon_{s h}$. This description is motivated by the fact that the sheath is a region depleted of electrons (similar to vacuum), as it was done in (D'Ippolito \& Myra 2006). The dielectric constant is of the order of $\varepsilon_{s h} \sim 1$, while $\varepsilon_{\|}$in the SOL is highly negative. However, in the presence of high power waves, the real sheath width is subject to large amplitude RF oscillations around its mean value. This may affect the effective sheath capacitance. Within these simple assumptions, the RF-SBC is linear and reads as (D'Ippolito \& Myra 2006)

$$
E_{t}^{p l}=\nabla_{t} V_{R F}=\nabla_{t}\left(\delta D_{n}^{p l} / \varepsilon_{s h}\right)
$$

Here, $E$ is the RF electric field, $D=\bar{\varepsilon} E$ is the RF electric displacement. RF quantities are evaluated at the plasma side of the sheath/plasma interface (superscript $p l$ ), where $D$ is continuous and $E$ exhibits a jump, because the dielectric tensor $\bar{\varepsilon}$ changes abruptly. The subscripts $n$ and $t$ refer, respectively, to the direction that is locally normal (towards plasma) and transverse to the wall. The sheath width $\delta$ is allowed to vary spatially, and its radial/poloidal distribution needs to be subsequently determined self-consistently from the DC sheath potential. Assuming that the magnetic field line follows the normal incidence, the SBC expression can be simplified and, for the sheath width provided by the Child-Langmuir's law assuming a plane geometry and high RF field amplitude, can be written as:

$$
\begin{gathered}
E_{\|}=\varepsilon_{s h} V_{R F} / \varepsilon_{\|} \delta \text { with } \delta=\lambda_{e}\left(V_{D C} / T_{e}\right)^{3 / 4} \text { [sheath capacitance], } \\
i^{+}\left[1-\exp \left(\frac{V_{b}-V_{D C}}{T_{e}}\right)\right]=\overline{\bar{\sigma}}_{D C} \cdot \nabla_{n} V_{D C}, V_{b}=V_{f}+\ln \left[I_{0}\left(\left|V_{R F} / T_{e}\right|\right)\right] \text { [rectification], }
\end{gathered}
$$

$$
V_{R F}=0 \text { at both radial ends of lateral boundaries. }
$$

A detailed discussion on different possible improvements of the SBC description in terms of geometry can be found in (Colas et al. 2012). The numerical method used to solve this problem consists on an iterative process, which ensures the convergence only if we are close to the solution of the problem. In (Colas et al. 2012), a way to obtain the first guess is proposed, assuming that the RF electric fields are to saturate the current in the sheath called wide sheath regime. This wide sheath solution becomes independent of the DC potential, and such method allows to converge (Jacquot et al. 2014), which is not the case if you assume no sheath width for the first guess.

The main results are that the electric field of the slow wave can be drastically changed, and the SBC behaviour can go from metallic conditions to transparent boundary conditions, depending on the plasma parameter and on the amplitude of the RF potential. This means that the perpendicular DC conductivity is a crucial parameter to determine the radial extension of the DC potential, thus (impacting / explaining / leading to) the power losses and spurious effects induced by the energetic ion flux on the wall (impurity injection, hot spots). The experimental results obtained with the new Faraday screen of Tore Supra ICRH antenna (Jacquot et al. 2014) show that further work has to be done to obtain a relevant modelling for any ITER scenarios, for example, the improvement of the RF sheath at oblique incidence developed in (Kohno et al. 2012).

To reach the goal with a predictive tool which is relevant for ITER and fusion reactor, some physical issues have to be studied, such as: (i) the role of radial particle turbulent transport on the RF sheath properties (this part can be important in the case of oblique $\mathrm{RF}$ sheath near the grazing angle to determine the DC potential drop as a function of the 
RF potential amplitude and particle turbulence flux level); (ii) the secondary emission is also an important parameter to determine the RF sheath properties; (iii) since the $\mathrm{RF}$ sheath takes place in the neighbouring of the wall or antenna structure, the role of recycling has to be studied, which also allows to refine the knowledge on the contributions of a ion species mixture or dusts to the RF sheath properties. These tasks will have an answer in the framework of the EURO fusion Enabling Research project devoted to the simulation of the ICRH heating including the RF sheath Physics.

The knowledge of the total flux, coming from the integration of all the fluxes reaching the wall at any titling angle of incidence, associated to the rectified potential, is a key point for the safety of the ICRH antenna design. Simulations can provide crucial information on this aspect, in the case we are able to generate particle turbulent flux with the relevant properties, that are included in the model. There are a few possibilities: (i) to implement an RF antenna in a turbulent code (seems too complex); (ii) to have a kinetic simulation (including all the system within the condition) to simulate the turbulence; or (iii) to introduce a term in the equation of motion able to produce a random walk with an average velocity non-equal to zero, which can be associated to turbulent transport. Including dissipation, the natural model corresponds to the Langevin's equations, instead of the motion equations in PIC codes. Such operator induces a global particle flux, keeping constant the energy of the system. However the number of particles required is so high that an accurate parallel computing is needed especially for ITER or further fusion reactors. That is, if we want to have a predictive tool, since we have to combine different phenomena (including macroscopic changes of the plasma target) during the heating, until we reach an asymptotic state. For such studies, the computation should definitively cover the overall plasma (including the interaction of a turbulent plasma with the wall (Kuhn et al. 2007)), because the RF sheath properties depend on the species contained and, eventually, on accelerated dusts in the RF sheath (Ticos et al. 2012) if we want to estimate the life-time of an antenna structure in a fusion reactor.

\section{Role of density fluctuations on wave heating}

\subsection{Context}

Nowadays, the role of the density fluctuations is reconsidered, because the numerical tools as full-wave codes are now able to investigate their effects due to the improvement of the stability of numerical scheme and their performance using High Performance Computers (da Silva et al. 2015). We can also add the displacement of the cut-off layer on the IRCH antenna-plasma coupling (Clairet et al. 2004; Jacquot et al. 2013) (which had not been taken into account until now), as well as the depolarisation induced by the density fluctuations in fusion devices. In general, the depolarisation mechanism should be studied in detail, by itself, applied to fusion plasmas, in order to evaluate the impact on the wave heating or on current drive efficiency. In addition, as shown in (Peysson \& Decker 2014), the energy deposition is affected by the density fluctuations at the plasma edge changing the radiation pattern of the antenna. It can also explain the spectral gap if the wavenumber spectrum contains the specific range of parallel wavenumber values. This types of studies can have a big impact especially on the neoclassical tearing mode control by electron cyclotron current drive where the beam properties requirements are strong (Comisso \& Lazzaro 2010) and particularly when a narrow beam is needed (knowing that the density fluctuations at the plasma edge induces a broadening of the launched Gaussian beam as in ITER cases (Sysoeva et al. 2014, 2015; Peysson \& Decker 2014)). In these works, the depolarisation effect is not taken into account and has not 
been studied in detail, even though it has been shown that the magnetic shear in a tokamak plays no role (Mazzucato 1998). This phenomenon is known in other domains of telecommunication (Tenouxa \& Lostanlen 2012), space plasma Physics (Jandieri et al. 2011) or laser plasma Physics (Javan \& Adli 2013), and only recently in fusion in (Mirnov et al. 2014). The polarisation changes have been studied in Astrophysics (Ledenev et al. 2002). The tools developed in these domains can be useful to study the full impact of the turbulence on the wave launched to heat the fusion plasmas. The simulation of the impact of the turbulence on the wave propagation requires full-wave codes, in order to take into account multi-scattering inducing beam broadening, multi-reflection able to modify the polarisation, and wave-trapping able transiently to enhance the heating power due to local enhancement of the wave amplitude (Heuraux et al. 2010). However, to integrate all the phenomena, a full-wave code has to describe all the components of the wave electric fields at least into two directions (radial, poloidal or toroidal). A 3D simulation (no mode decomposition) over realistic space domain of ITER or DEMO is too demanding in terms of computation resources at present. We now detail how to proceed in order to build a full-wave simulation to compare theory and numerical results for beam broadening induced by density fluctuations at the plasma edge (in the case of electron cyclotron resonant heating or current drive using O-mode or X-mode), and look at the main results for ITER cases.

\subsection{Beam broadening simulation, from numerical scheme to results in the electron cyclotron frequency range}

Before simulating such Gaussian beam broadening induced by density fluctuations, we have to choose between different options, bearing in mind the problem to solve the study of the beam shape evolution as a function of the radial coordinate for a given turbulence with known characteristics (wavenumber spectrum, density fluctuations profile) for a given set of plasma parameters (magnetic field, density profile, temperature profile). The choices are explained by answering to questions concerning numerical schemes, parallel computing, stability before presenting the results on beam widening. The inputs can come from measurements in experiments, provided by equilibrium and turbulence codes, or built from analytical expressions. The properties of the launched wave beam including

the polarization (O-mode or X-mode here) can be idealized through the expression of a Gaussian beam, or computed using a complete description of the launching system (wave guide, mirror, horn). In possession of all the required data, we have to define the computational domain and the properties of the border. That is to say, the boundary conditions [Perfect Electric Conductor (PEC), PML, partial Absorbing Boundary Condition $(\mathrm{ABC})$, UTS or not for the wave launcher] as a function of the assumptions (single-pass absorption here) or imposed by the knowledge of the plasma properties (with or without mode conversion). At this stage, one needs to choose the numerical scheme, which depends on different options and physical phenomena that must be considered. Here, the options are: (i) ergodic treatment associated with an average of the wave intensity overall samples of turbulence matrices (Helmholtz, frequency domain (Heuraux \& da Silva F. 2012)); or (ii) time averaging of the wave intensity (FDTD(Taflove \& Hagness 2000)) for a time-dependent turbulence matrix. It is clear that numerical diagnosis have to be defined at the same time as a function of the studied parameters (wave intensity and poloidal correlation of the wave intensity) and as the control parameters (wave energy conversion, stopping criterion etc). For this last part, a minimal knowledge of the computation parameters is necessary, as are the required number of time steps, computation time per time step, memory requirements, among others. A parallel computing strategy has to be defined for the implementation (determining the algorithm), assuming this nu- 
merical scheme is stable and meets the imposed criteria on accuracy, energy conservation, and others constraints. At the end, if it is necessary in the case of a long run, a possible strategy is to dump the data according to the storage possibilities.

Now, we go in the detail to answer the question: Why do we have to choose a timedependent algorithm for this study? The choice is first driven by the physical constraints. We want to describe the $\mathrm{O}$ and $\mathrm{X}$ modes in the same plasma conditions. In the frequency domain, the Helmholtz solver for the $\mathrm{X}$-mode is more problematic than the O-mode, because a set of coupled partial differential equations containing cross-derivatives has to be solved, which introduces numerical difficulties. Therefore, in the frequency domain, we have two codes to implement. One of the codes (for X-mode) contains numerical difficulties, without the possibility to introduce a mode conversion without writing another, even more complex, code. A Maxwell's equation FDTD solver allows treating both polarizations with the same algorithm, and only the source term has to be defined, by changing the excited wave fields. The second point concerns the choice of the time averaging. Knowing that the turbulence has its own correlation time $\tau_{c}$, the macroscopic behaviour of the wave is exhibited when the time averaging is greater than $\tau_{c}$, in practice, at least $4 \tau_{c}$. Maxwell's equations solver also has the advantage of extending to other applications without changing its kernel, even if the computation time is longer than a dedicated code. After choosing Maxwell's equation solver, a second question quickly arises: How to generate the turbulent matrix? The standard method corresponds to a sum of trigonometric functions fulfilling the prescribed correlation time and wave number spectrum of the turbulence. The direct summation of modes is irrelevant, due to the time required to compute a turbulence matrix. The use of Fast Fourier Transform is the fastest way to address this, associated to the random phase between each mode (da Silva et al. 2010). With such method, it is possible to introduce a phase relationship in time that can mimic a dispersion relation or a specific turbulence behaviour. Here the goal is to obtain an average value of the wave intensity at the asymptotic state, a way to speed-up the computation is to generate a bigger turbulence matrix extended in poloidal direction (keeping the imposed wave number spectrum), and to move it at a given constant speed (less than $10 \%$ of the speed of light) in the poloidal direction (da Silva et al. 2006b). The price to pay is the storage of a huge turbulence matrix that introduces limitations, depending on the computation resources. Another constraint can appear for the X-mode: the number of time steps needed before the birth of a numerical instability associated with a high level of the turbulence. This numerical instability is described in (da Silva et al. 2015), as are the possibilities to remove it. An important point to mention is that the numerical scheme used should preserve the energy of the system, in order to conserve the statistical properties we want to study. This kind of algorithm is presented in (da Silva et al. 2015). To avoid a huge storage, the diagnostic is defined on lines at constant radial values (assuming that the beam spatial evolution is small between each line). This can be optimized to speed up the computation and reduce data storage. As a Gaussian beam is used, it allows evaluating the radial distance between each of the diagnosis line. An unexpected problem takes place the number of time steps necessary to obtain an asymptotic state for the wave intensity (more than $1.510^{7}$ time steps for an O-mode case and twice as more for X-mode) which is too heavy to compute it in a single run. Thus, the choice was to average over time, more than one million time steps, on 20 samples of turbulence matrix. The total computation time is approximately one week on a single processor. It is clear in such cases that parallel computing is required.

Another question is What is the best choice for the parallel computing, MPI or/and 
OpenMP communication tools? In our case, a parallel version of the full-wave FDTD code REFMULX was developed within the framework of a High Level Support Team (HLST) project. One of the dimensions of the bi-dimensional domain was decomposed, using the MPI standard in such a manner as to maximise the contiguousness of the code's data representation in memory. The solver routines were modified accordingly, to allow each MPI task to work on a different sub-domain. The coupling between the neighbouring sub-domains (fixed by the scheme's numerical stencil) was ensured by the usage of ghost-cells, which store the data that need to be exchanged via standard point-to-point MPI calls. Following the trend of modern hierarchical architectures on which multiple cores share memory, a threaded-based parallelism was also implemented in the same direction of REFMULX's domain. Such a hybrid implementation allows several threads to exist within each sub-domain (i.e. MPI task). Since the threads share the same memory space, there is no need for inter-node communication nor guard-cells, typically leading to better memory usage at no performance cost. This expectation was confirmed with the parallel REFMULX code, whose scaling measurements on HELIOS-CSC supercomputer in Japan showed a speedup of two orders of magnitude on a couple of hundred cores for a typical 1500x1000 grid-count case, compared to the original serial version. Beyond this point, there is not enough work-per-core to justify the usage of further resources. However, bigger problems (e.g. an ITER case) provide that extra computational work. In other words, new physics studies are potentially enabled by the parallel version of REFMULX. Additionally, in light of the results obtained, extending the modelling to three spatial dimensions becomes now feasible, since all the parallelization techniques here developed shall be directly deployed in that case. Since the grid-count increases by two, or even three orders of magnitude, further extending the domain decomposition might be required. However, it is already clear that some effort needs to be put into implementing parallel I/O in the code, which was already measured to be a bottleneck in the biggest two-dimensional test-cases ran so far.

To improve the stability of our code, a smoothing of the turbulence matrix is done just at the plasma edge to avoid stiff index gradients. It is also amazing to see that the optimization done during the parallelization of the code has also improved the code stability. The code remains stable above the turbulence level expected in ITER case. Knowing that the code here has to run in unknown conditions as it is for DEMO or for the data coming from a given turbulence code. Cases in which the turbulence level is higher than the threshold of the numerical instability (NI) should trigger a warning. In addition, the turbulence is rescaled below the threshold and the wanted results are extrapolated knowing the non-linear dependencies. The NI threshold value is high enough to evaluate all the non-linear phenomena found previously, e.g. the non-linear Bragg backscattering (Gusakov et al. 2009) or the wave trapping (Heuraux et al. 2010; Gusakov et al. 2011). The possibility to have non-linear effects is an experimental evidence in the Stellerators through the measurements of Bragg backscattering (Batanov et al. 2013).

To illustrate beam broadening, ITER-like plasma parameters are used as inputs, including all the possible inhomogeneities described by the theoretical models presented in (Sysoeva et al. 2015), and those associated to electron cyclotron heating or current drive. The frequency is taken equal to $170 \mathrm{GHz}$. The density fluctuations (the RMS value), the density profiles and the turbulence perpendicular wavenumber spectrum used in the analysis, are presented in Fig.3. The spectrum density, which corresponds to a rescaled wavenumber spectrum coming from experimental data (Gerbaud et al. 2006), is in agreement with GYRO simulations (Casati et al. 2009). The radial evolution of 

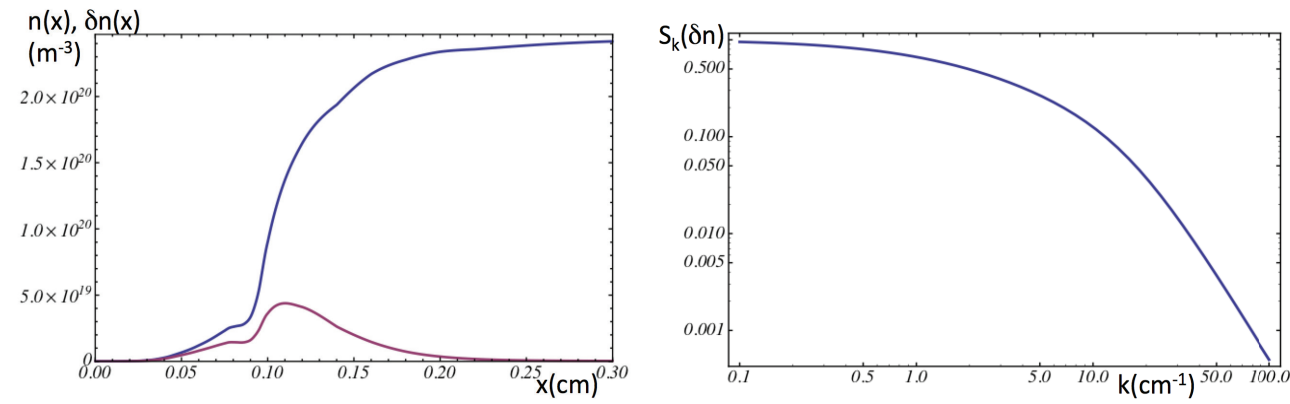

FIgURE 3. Inputs for an ITER case: (left) density and density fluctuations profiles, and (right) wavenumber spectrum of density fluctuations identical in $\mathrm{x}$ and $\mathrm{y}$ direction

beam width shows the role of the turbulence on the beam divergence changes (see Fig.4 (right)), and the enhanced divergence after the crossing of the turbulence zone. The little inflection of the beam width for the highest level of turbulence corresponds to the fact that part of the beam enters into the PML, showing that we have to be careful when numerical tools are applied automatically. The snapshot of the wave electric field shows that turbulence breaks completely the wave-front, gives a filamentary structure to wave beam and spreads the wave intensity (see Fig.4). After averaging over a very long time $(\sim 3 \mu \mathrm{s})$ compared to the time of flight (few ns), it is amazing to see that the wave intensity recovers a Gaussian beam shape, but with a wider angular divergence. This lack of directivity can be reduced if the magnetic field is increased for the same density profile or the density at the center is reduced. In this case, for a fusion reactor, it will be better to work at higher magnetic field intensities. Looking at the computation time [re-scaled into real-time (few $\mu$ s)] that is necessary to obtain a Gaussian beam (on average), it is much less than the time necessary to measure the wave intensity ( $\sim \mathrm{ms})$ experimentally. This fact can explain why the modelling seems to work, but not with the adequate or expected parameters. This is simply due to the omission of the effect on wave propagation of plasma edge turbulence that drives the use of the Gauss beam parameters evaluated in vacuum, instead of the ones that consider the density fluctuations effects. The role played by the wavenumber spectrum characteristics is important (see (da Silva et al. 2010)). The dependency in poloidal wavenumber is detailed in (Sysoeva et al. 2015). Thus, the knowledge of the density fluctuations characteristics is required to evaluate these effects, which is not really the case for ITER and even less so for DEMO. Therefore, in future, we need to have a measurement of the turbulence characteristics in real-time, to be able to work with the wave heating or current-drive systems in an optimal way.

In conclusion, the control of the growing magnetic islands associated with neoclassical tearing modes can be less easy due to the beam broadening (Comisso \& Lazzaro 2010). However, tools are now available to evaluate the corrections that need to be introduced in a ray tracing code after the crossing of a high level of turbulence at the plasma edge. The full-wave code was used in (Sysoeva et al. 2015) to validate the theoretical models. One can notice that the full-wave simulation is able to precise the upper limit of the turbulence level for which the theoretical models are applicable. It is easy to see that, for such studies, the interplay between different codes is important. A turbulence code will be interesting for providing the turbulence characteristics, especially the poloidal wavenumber spectrum and the density fluctuations profile. However, the real-time use of the turbulence code data in the full-wave code is risky. The risk stems from the fact that, usually, an interpolation is necessary or a smoothing should be applied. To have 

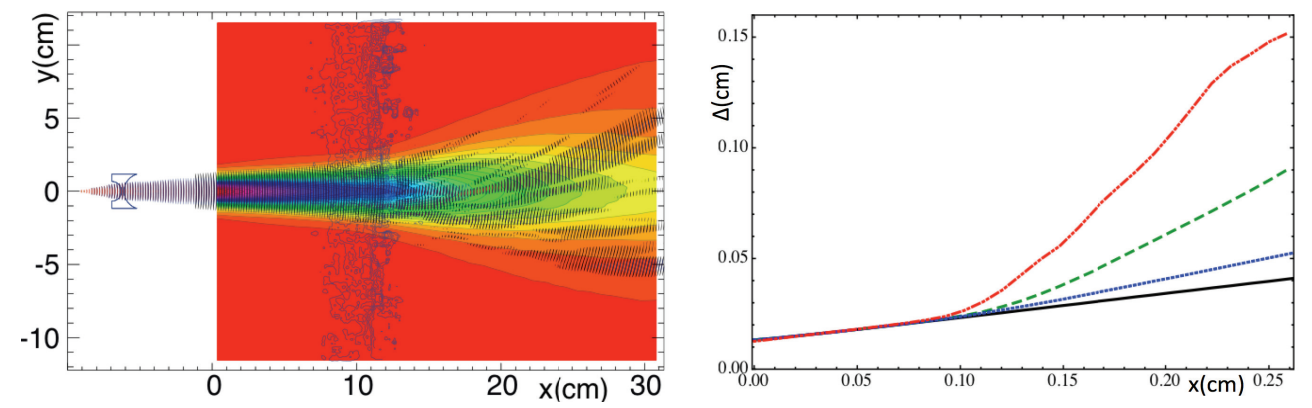

Figure 4. (left) Contour plots superimposition of a snapshot of the wave electric field positive part over the averaged intensity in an ITER case and of the density profiles in the turbulent zone; and (right) spatial evolution in the plasma zone of the quasi-Gaussian beam width in vacuum (black), in plasma w/o turbulence (blue dot), in turbulence $\delta n=3 \%$ (green dash) and in turbulence $\delta n=5 \%$ (red dots-dash)

a universal tool to adapt the synthetic data coming from a turbulence code, without changing the properties of the turbulence, is not an easy task. This kind of method is, at present, turbulence code dependent, probably due to the different boundary conditions of these codes (flux or gradient). The use of a full-wave code can be limited to the plasma edge area, in order to determine the beam properties inside the plasma at a given position for which the density fluctuations level is low enough to keep the beam characteristics constant. Above this position, beam ray tracing can be used (Figini et al. 2012) to determine the energy deposition volume.

The role of turbulence at the plasma edge has to be taken into account for any wave heating system, because of changes induced on: (i) the RF sheath boundary conditions surrounding the antenna structure in the ion cyclotron frequency range due to the sensitivity of coupling as function of the cut-off position (Clairet et al. 2004); (ii) the effective wavenumber spectrum launched into the plasma by the lower hybrid grill (Peysson \& Decker 2014); and (iii) the beam properties in the electron cyclotron frequency range Sysoeva et al. (2015).

\section{Conclusions and new developments}

Over the past year, the simulations on wave heating in fusion plasmas have been widely improved. They started being integrated in a platform (e.g. ITM platform (Falchetto et al. 2014)) able to provide the changes of the macroscopic plasma parameters induced by wave heating or current-drive. Following an iterative process, we can generally have access to an asymptotic state that allows predicting the behaviour of an experiment. Even if the computational resources increase (allowing to reach 3D description), important parts of the physical mechanisms are missing, crudely described or treated independently. Knowing the start-up phase of the stellarator W7X, the description of the wave propagation in the stellarator configurations should be rapidly improved, which is not obvious without making crude assumptions (Jucker et al. 2011). Recently, the role of density fluctuations has been reconsidered (as mentioned in (Peysson \& Decker 2014)), and the impact of turbulence is being actively studied. However, these works ignore the depolarization processes because they are negligible in quiet magnetized plasmas (even if there is magnetic shear (Mazzucato 1998)); or the wave reflection is always considered as perfect without changing the polarisation, which can be far from real conditions as shown in another 
domain (Bai et al. 2014). Nevertheless, in turbulent plasmas, the picture can be different from space plasmas (Jandieri et al. 2011) or laser plasmas (Javan \& Adli 2013). Therefore, many questions have to be addressed, such as What is the full role of the turbulence on the coupling and on the depolarisation, especially when the plasma flows intermittently? What is the role of single dense events? How does the time-averaging introduced by the coupling system acts on it? Is it relevant to consider only the averaged values of the plasma parameters integrated over the time-integration of the coupling system? In other words, Where is the effective position of the cut-off layer in turbulent plasma (considering the second order correction of the effective index associated to the density fluctuations (da Silva et al. 2003)) to consider for the evaluation of the coupling resistance? Is a model based on an effective index introducing a constant correction at the second order relevant for ICRH? A similar question on the accessibility condition and conversion layer has just now a response on the impact of the turbulence on O-X mode conversion (Popov 2015), which is part of the O-X-B heating scheme (Laqua et al. 1997). At present, for ICRH, the SBC condition description becomes more and more sophisticated. However, it stays crude, especially in the oblique case, where the magnetic field intercepts the wall far from the normal incidence. 1D PIC simulations at grazing incident angles show that the time scale to reach the equilibrium is too long, and a perpendicular transport as the recycling and ionization have to be taken into account to determine the RF sheath properties that are useful for SBCs. The ponderomotive effects have been evaluated considering the injected power density into the plasma. However, the crude expressions of the ponderomotive force, the disregard of the wave polarisations role in its expression, and the essential role of the quasi-neutrality, compensate its effects (Heuraux et al. 1994). Other non-linear effects are present, e.g. the parametric instability shown in (Gusakov et al. 2014), which need to be considered for a relevant description of a wave Heating System.

A numerical effort should be made to build a fast unconditional stable scheme that is able to preserve the energy of the system for all possible modes one can have in a time-dependent magnetized turbulent plasma. That is to say we must have an improved perfect matching layer or absorbing boundary conditions for the relevant modes, which is not the case at present (Jacquot et al. 2013). The SBC description should be improved and valid, at any angle. A way to cross the wave resonance (the real part of the index going to infinity) should be found without an artificial damping. Right now, we are far from this goal. In addition, this wave propagation code should integrate a coupling code, or have a full-geometrical description of the antenna structure able to support the induced currents on the antenna structure, in order to describe self-consistently the wave emission and the propagation of the emitted wave. No development has been made so far on the self-consistent numerical description of a heating system (ICRH, or LHCD) going from the backside of the antenna to the plasma core mainly due to too large computational resource needs. That is to say, no integration of a coupling antenna-plasma code into a wave propagation code describing the plasma heating (which should also integrate the macroscopic plasma parameter modifications) took place.

The use of the sub-domain decomposition should be generalized, since the true need of a full-wave code takes place in a limited area. To finalize, this code should be part of an integrated modelling framework, as it is planned for the ERCC 3D code that is under development (Coelho et al. 2013); the role of the transport through the evolution of the plasma parameters should be considered; and access to turbulence properties in a self-consistent description must be available. These can be solved properly only if the problem of data exchange is resolved adequately; knowing that, for numerical constraints, the meshes in each module are different, and thus a coherent interpolation should be done. 


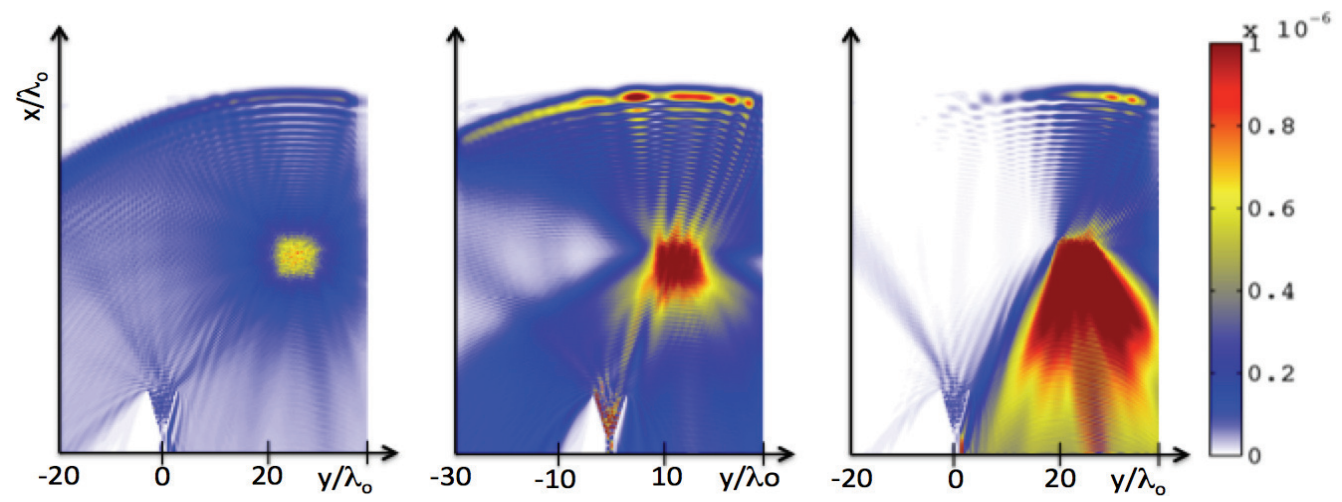

Figure 5. Contour plots of the modulus of the electric field difference between perturbed and unperturbed density profile averaged over 200 turbulence matrix samples (scattered wave in Born approximation); (left) in the case of a wavenumber spectrum with a wide range of k-values covering all the possible scattering process; (centre)same as left case but for intermediate range of $\mathrm{k}$, for which the radiation pattern presents a hole; and (right) same case with a very narrow spectrum around zero, able to induce forward scattering only $k<k_{\text {Airy }}$; and in all cases, the density fluctuations amplitude is $\delta d n / n_{c}=0.1 \%$.

However, it is necessary to be aware that the spectra can be truncated or distorted in this interpolation, or intrinsic, due to the mesh choice. For instance, spatial steps that are too wide are used in turbulence codes to describe properly the small spatial scales involved in the scattering processes really present in an experiment, e.g. in back scattering (Batanov et al. 2013). That is to say, for the turbulence code to be relevant, it should include such possibilities, by choosing a spatial grid able to precisely describe these phenomena, which could be incompatible with the numerical constraints. Three different cases are shown on Fig.5 just to illustrate this purpose of the dependencies on the scattered field as a function of the turbulence spectrum. In the first case, all spatial scales are present, and the scattered is more or less isotropic after averaging. Then, part of the spectrum is truncated and, as a consequence, an anisotropy appears in the scattered field pattern, according to the Bragg rule (see (Fanack et al. 1996)). In the last one, only forward scattering is possible, showing a narrow lobe for the scattered wave emission. Therefore, this shows that the simulation, in order to be relevant, has to take care of the scales in time and space present in an experiment, which is not always possible, mainly due to the limitations of available numerical resources. As a conclusion the simulations on wave heating help us to improve the heating systems when we add more Physics in it, as this was shown, partially.

Acknowledgements: The authors acknowledge the support of ANR under contracts ANR-12-BS01-0006-01, ANR-12-BS09-0028-02, ANR-12-JS09-0013-01. Moreover, this work was carried out within the framework of the European Fusion Development Agreement and the French Research Federation for Fusion Studies. A part of this work was carried out using the HELIOS supercomputer system at the Computational Simulation Centre of the International Fusion Energy Research Centre (IFERC-CSC), Aomori, Japan, under the Broader Approach collaboration between Euratom and Japan, implemented by Fusion for Energy and JAEA. This project has received funding from the European Unions Horizon 2020 research and innovation programme, under grant agreement number 633053. IST activities also received financial support from ?Fundação para a Ciência e Tecnologia? through project Pest-OE/SADG/LA0010/2013. It is supported by the European Communities under the contract of Association between Euratom and CEA. The 
views and opinions expressed herein do not necessarily respect those of the European Commission.

\section{REFERENCES}

Artaud, J.F., Basiuk, V., Imbeaux, F. \& Schneider, M. ET al 2010 The cronos suite of codes for integrated tokamak modelling. Nuc. Fusion 50, 043001.

BAI, B., Li, X. \& LiU, Y. ET AL 2014 Effects of reentry plasma sheath on the polarization properties of obliquely incident em waves. IEEE Trans. Plas. Sci 42, 3365-3372.

Batanov, G. M., Borzosekov, V. D., Kovrizhnykh, L. M. \& Kolik, L. V. et al 2013 Backscattering of gyrotron radiation and short-wavelength turbulence during electron cyclotron resonance plasma heating in the l-2m stellarator. Plasma Phys. Reports 39, 444455.

BÉRENGER, J.-P. 1994 Numerical modeling of the coupling of an icrh antenna with a plasma with self-consistent antenna current in the ion cyclotron range of frequencies. J. Comput. Phys. 114, 185-200.

Bertelli, A., Maj, O., Poli, E., Harwey, C.W. \& et al. 2012 Paraxial wentzelkramersbrillouin method applied to the lower hybrid wave progation. Phys. Plasmas 19, 082510.

Bobkov, V., Braun, F., Dux, R. \& Herrmann, A. et al 2010 Assessment of compatibility of icrf antenna operation with full $\mathrm{w}$ wall in asdex upgrade. Nuc. Fusion 50, 035004.

Bonoli, P. 1985 Linear theory of lower-hybrid wave in tokamak plasmas. In Wave heating and current drive in plasmas, pp. 175-218. Gordon and Breach New York.

Bonoli, P.T. 2014 Review of recent experimental and modeling progress in the lower hybrid range of frequencies at iter relevant parameters. AIP Conference Proceedings 1580, 15-24.

Bornatici, M., Cano, R., De Barbeiri, O. \& Englemann, F. 1983 electron cyclotron emission and absoprtion in fusions plasmas. Nuc. Fusion 23, 1153-1257.

Brambilla, M. 1999 Numerical simulation of ion cyclotron waves in tokamak plasmas. Plasma Phys. Cont. Fusion 41, 1-34.

Brambilla, M. \& Bilato, R. 2009 Advances in numerical simulations of ion cyclotron heating of non-maxwellian plasmas. Nuc. Fusion 49, 085004.

Budny, R.V., Berry, L., Bilato, R., Bonoli, P. \& et Al 2012 Benchmarking icrf full-wave solvers for iter. Nuc. Fusion 52, 023023.

CAIRns, R.A. 1991 In Radiofrequency heating of Plasmas, pp. Ch3-Ch5. Adam Higer, IOP Publishing.

Campos Pinto, M., Sonnendrücker, E., Friedman, A., Grote, D.P. \& Lund, S.P. 2014 Noiseless vlasovpoisson simulations with linearly transformed particles. J. Comp. Phys. 275, 236-256.

Casati, A., Grangirard, V., Bourdelle, C. \& Hennequin, P. et al 2009 Turbulence in the tore supra tokamak measurements and validation of nonlinear simulations. Phys. Rev. Lett. 102, 165005.

Clairet, F., Colas, L., Heuraux, S. \& Lombard, G. 2004 Icrf coupling and edge density profile on tore supra. Plasma Phys Cont Fusion 46, 1567-1581.

Comlho, R., Akaslompolo, S., Dinklage, A. \& Kus, A. Et al 2013 Synthetic diagnostics in the eu-itm simulation platform. Fusion Science and Techno. 63, 1-9.

Colas, L., Jacquot, J., Heuraux, S. \& Faudot, E. ET Al 2012 Self consistent radiofrequency wave propagation and peripheral direct current plasma biasing: Simplified three dimensional non-linear treatment in the wide sheath asymptotic regime. Phys. Plasmas $\mathbf{1 9}$, 092505.

Colas, L., Zou, X. L., Paume, M., Chareau, J.-M. \& et al 1998 Internal amgnetic fluctuations and lectron heat transport in tore supra. Nucl. Fusion 38, 903-918.

Comisso, L. \& Lazzaro, E. 2010 Two-dimensional effects in the problem of tearing modes control by electron cyclotron current drive. Nuc. Fusion 50, 125002.

Courant, R., Friedrichs, K. \& Lewy, H. 1928 Uber die partiellen differenzengleichungen der mathematischen physik. Mathematische Annalen 100, 32-74.

Crouseilles, N., Lemou, M. \& MÉhats, F. 2013 Asymptotic preserving schemes for highly oscillatory vlasovpoisson equations. J. Comp. Phys. 248, 287-308. 
Decker, J., Peysson, Y. \& Coda, S 2012 Effect of density fluctuations on eccd in iter and tcv. Eur. Phys. J. Web Conf. 32, 01016.

Després, B., ImBert-GÉrARD, L.-M. \& Weder, R. 2014 Hybrid resonance of maxwell's equations in slab geometry. Journal de Math. Pures et Appliquées 101, 623-659.

D'Ippolito, D. A. \& MyrA, J. R. 2006 A radio-frequency sheath boundary condition and its effect on slow wave propagation. Phys.Plasmas 13, 102508.

Dumont, R. J. \& Zarzoso, D. 2013 Heating and current drive by ion cyclotron waves in the activated phase of iter. Nucl. Fusion 53, 013002.

VAN Eester, D. \& KoCH, R. 2001 Integrating the finite-temperature wave equation across the plasma/vacuum interface. Plasma Phys. Cont. Fusion 43, 779-794.

EesteR, D. VAN 2012 Modeling particle and current drive in fusion machines: Brief review of adopted techniques. Transaction on Fusion Science and Technology 61, 347-354.

Falchetto, G.L., Coster, D., Coelho, R. \& Scott, B.D. Et Al 2014 The european integrated tokamak modelling (itm) effort: achievements and first physics results. Nuc. Fusion 54, 043018.

Fanack, C., Boucher, I., Heuraux, S., Leclert, G., Clairet, F. \& Zou, X.L. 1996 Ordinary mode reflectometry: modifications of the backscattering and cut-off responses due to shape of localized density fluctuations. Plasma Phys. Cont. Fusion 40, 1915-1930.

Faudot, E., Heuraux, S. \& Colas, L. 2006 Peaking criterion for rectified potential in front of icrf antennas in fusion plasma. Phys. Plasmas 13, 042512.

Faudot, E., Heuraux, S., Colas, L. \& GunN, J. P. 2010 Broadening of rectified potential structures induced by rf currents in a magnetized plasma application to iter scrape-off-layer. Phys. Plasmas 17, 042503.

Faudot, E., Heuraux, S., Kubic, M., Gunn, J. \& Colas, L. 2013 Fluid modeling of rf and dc currents in a biased magnetized plasma. Phys. Plasmas 20, 043514.

Figini, L., Decker, J., Farina, D., Marushchenko, N. B., Peysson, Y., Poli, E., WestERHOF, E. \& CONTRIBUTORS, ITM-TF 2012 Benchmarking of electron cyclotron heating and current drive codes on iter scenarios within the european integrated tokamak modelling framework. EPJ Web of Conferences 32, 01011.

Fuchs, V., Ram, A.K., Schultz, S.D., Bers, A. \& Lashmore-Davies, C.N. 1995 Mode conversion and electrons damping of the fast alfven wave in a tokamak at ion-ion 16371647.

Gerbaud, T., Clairet, F., Sabot, R. \& Sirinelli, A. et al 2006 Comparison of density fluctuation measurements between o-mode and x-mode reflectometry on tore supra. Rev. Sci. Instrum. 77, 10E928.

GNESIN, S. 2011 Electron cycltron heating and suprathermal electron dynamics in the tcv tokamak. PhD thesis, EPFL Laussane.

Gusakov, E.Z., Heuraux, S., Irzak, M. \& Popov, A. Yu. 2011 Possibility of giant scattering enhancement due to wave trapping in a reflectometry experiment. Physica Scripta 84, 04504 .

Gusakov, E.Z., Heuraux, S. \& Popov, A. Yu. 2009 Nonlinear regime of bragg backscattering leading to probing wave trapping and time delay jumps in fast frequency sweep reflectometry. Plas. Phys. Cont. Fusion 51, 065018.

Gusakov, E.Z., Popov, A.Yu. \& Saveliev, A.N. 2014 Trapping of electron bernstein waves in drift-wave eddies and parametric decay instability at second harmonic ecrh in toroidal devices. Plasma Phys. Control. Fusion 56, 015010.

Heuraux, S., Faudot, E., da Silva, F., Jacquot, J., Colas, L., Hacquin, S., Teplova, N., Sysoeva, E.V. \& Gusakov, E.Z. 2014 Study of wave propagation in various kinds of plasmas using adapted simulation methods, with illustrations on possible future applications. C. R. Physique 15, 421-429.

Heuraux, S., Gusakov, E.Z., Popov, A.Yu, da Silva, F. \& Irzak, M. 2010 Simulations on the role of the resonance of the probing wave on reflectometry measurements in fluctuating plasmas. IEEE Trans. Plas. Sci 38, 2150-2158.

Heuraux, S., Lelcert, G. \& Hadjoudj, Y. 1994 Low-frequency responses of a plasma to a large amplitude high-frequency wave polarized on the extraordinary mode. Journal de Physique III 4, 839-848.

Heuraux, S. \& DA Silva F. 2012 Simulations on wave propagation in fluctuating fusion plasmas 
for reflectometry applications and new developments. Discrete and Continuous Dynamical System Series S 5, 307-328.

IrZAK, M. A. \& Popov, A. Yu 2008 2d modeling of the o-x conversion in toroidal plasmas. Plasma Phys. Control. Fusion 50, 025003.

JACKson, J. D. 1999 Classical Electrodynamics. In Classical Electrodynamics (ed. JohnWiley \& sons), pp. 19-22.

Jacquet, P., Colas, L., Mayoral, M.-L. \& Arnoux, G. et Al 2011 Heat-loads on jet plasma facing components from icrf and lh wave absorption in the sol. Nuc. Fusion 51, 103018.

Jacquot, J., Colas, L., Clairet, F., Goniche, M., Heuraux, S., Hillairet, J. \& LomBARD, G. Milanesio, D. 2013 2d and 3d modeling of wave propagation in cold magnetized plasma near the tore supra icrh antenna relying on the perfectly matched layer technique. Plasma Phys. Control. Fusion 55, 115004.

Jacquot, J., Milanesio, D., Colas, L., Coore, Y., Gunn, J., Goniche, M., Heuraux, S. \& Kubic, M. 2014 Radio-frequency sheaths physics: Experimental characterization on tore supra and related self-consistent modeling. Phys. Plasmas 21, 061509.

Jaeger, E.F., Bachtelor, D.B. \& Stallings, D.C. 1993 Influence of various physics phenomena on fast wave current drive in tokamaks. Nuc. Fusion 33, 179-195.

JANDieri, G. V., Ishimaru, A. \& JANDieri, V. G. ET AL 2011 Depolarization of metric radio signals and the spatial spectrum of scattered radiation by magnetized turbulent plasma slab. Prog. Electromag. Res. PIER 112, 63-75.

JaVAN, N. S. \& AdLI, F. 2013 Polarization effect on the relativistic nonlinear dynamics of an intense laser beam propagating in a hot magnetoactive plasma. Phys. Rev. E 88, 043102.

Jucker, M., Graves, J.P., Cooper, W.A., Mellet, N., Johnson, T. \& Brunner, S. 2011 Integrated modeling for ion cyclotron resonant heating in toroidal systems. Comp . Phys. Comm. 182, 912-925.

Kohno, H., Myra, J. R. \& D'ipolitto, D. A. 2012 Numerical modeling of the coupling of an icrh antenna with a plasma with self-consistent antenna current in the ion cyclotron range of frequencies. Comp. Phys. Comm. 183, 2116-2127.

Kuhn, S., Tskhakaya, D.D. \& Tskhakaya, (JR) D. 2007 The magnetized plasma-wall transition (pwt) and its relation to fluid boundary conditions. Comp. Phys. Comm. 177, 8083.

Kuley, A., WAng, Z.X., Lin, Z. \& Wessel, F. 2013 Verification of particle simulation of radio frequency waves in fusion plasmas. Phys. Plasmas 20, 102515.

Lancellotti, V., Milanesio, D., Maggiora, R., Vecchi, G. \& Kyrytsya, V. 2006 Topica: an accurate and efficient numerical tool for analysis and design of irrf antennas. Nuc. Fusion 46, S476-S499.

Laqua, H. P., Erckmann, V., Hartfuss, H. J. \& Laqua, H. 1997 Resonant and nonresonant electron cyclotron heating at densities above the plasma cutoff by $\mathrm{o}-\mathrm{x}-\mathrm{b}$ mode conversion at the w7-as stellarator. Phys. Rev. Lett. 78, 3467-3470.

Ledenev, V.G., Tirsky, V. V., Tomozov, V. M. \& Zlobec, P. 2002 Polarization changes in solar radio emission causedy scattering from high-frequency plasma turbulence. $A A \mathbf{3 9 2}$, 10891094.

Marushchenko, N.B., Turkin, Y. \& MaAssberg, H. 2014 Ray-tracing code travis for ecr heating, ec current drive and ece diagnostic. Comp. Phys Comm. 185, 165-176.

MazzucAto, E. 1998 Microwave reflectometry for magnetically confined plasmas. Rev. Sci. Instrum. 69, 2201-2217.

Milanesio, D., Meneghini, O., Maggiora, R., Guadamuz, S., Hillairet, J., LancelLOTti, V. \& VECCHI, G. 2012 Toplha an accurate and efficient numerical tool for analysis and design of lh antennas. Nuc. Fusion 52, 013008.

Mirnov, V.V., Brower, D.L., Hartog, D.J. \& Ding, W.X. ET Al 2014 Electron kinetic effects on interferometry, polarimetry and thomson scattering measurements in burning plasmas. Rev. Sci. Instrum. 85, 11D302.

Noterdaeme, J.-M. \& Oost, G. VAN 1993 The interaction between waves in the ion cyclotron range of frequencies and the plasma boundary. Plasma Phys. Controlled Fusion 35, 14811511.

Pécoul, S., Heuraux, S., Koch, R. \& Leclert, G. 2002 Numerical modeling of the coupling 
of an icrh antenna with a plasma with self-consistent antenna current. Comp. Phys. Comm. 146, 166-187.

Perkins, W. 1989 Radiofrequency sheaths and impurity generation by icrf antennas. Nuc. Fusion 29, 583.

Peysson, Y. \& Decker, J. 2014 Numerical simulations of the radio-frequency-driven toroidal current in tokmaks. Fusion Science and Technology 65, 22-42.

Peysson, Y., Decker, J. \& Morini, L. 2012 A versatile ray-tracing code for studying rf wave propagation in toroidal magnetized plasmas. Plasma Phys. Cont. Fusion 54, 045003.

Poli, F.M., Kessel, C.E., Bonoli, P.T., Batchelor, D.B., Harvey, R.W. \& Snyder, P.B. 2014 External heating and current drive source requirements towards steady-state operation in iter. Nuc. Fusion 54, 073007.

Popov, A. Yu 2015 Anomalous reflection of electromagnetic waves at o-x mode conversion in 2d inhomogeneous turbulent plasma. Plasma Phys. Control. Fusion 57, 025010.

Prater, R., Farina, D., Gribov, Yu. \& Harvey, R.W. et al 2008 Benchmarking of codes for electron cyclotron heating and electron cyclotron current drive under iter conditions. Nuc. Fusion 48, 035006.

da Silva, F., Campos Pinto, M., Després, B. \& Heuraux, S. 2015 Stable coupling of the yee scheme with a linear current model. J. Comp. Phys. 295, 24-45.

Da Silva, F., Heuraux, S., Gusakov, E.Z. \& Popov, A.Yu 2010 A numerical study of forward- and back-scattering signatures on doppler reflectometry signals. IEEE Trans. Plas. Sci 38, 2144-2149.

da Silva, F., Heuraux, S., Hacquin, S. \& Manso, M. 2005 Unidirectional transparent signal injection in finite-difference time-domain electromagnetic codes. J. Comput. Phys. 203, 467-492.

Da Silva, F., Heuraux, S. \& Manso, M. $2006 a$ Developments on reflectometry simulations for fusion plasmas : applications to iter position reflectometry. J. Plasma Phys. 72, 1205-1211.

Da Silva, F., Heuraux, S. \& Manso, M. $2006 b$ Studies on o-mode reflectometry spectra simulations with velocity shear layer. Nuc. Fusion 46, S816S823.

Da Silva, F., Heuraux, S., Manso, M.E. \& Varela, P. 2003 A 2D FDTD full-wave code for simulating the diagnostic of fusion plasmas with microwave reflectometry. In Computational methods in Engineering and science (ed. A.A Balkema), , vol. ISBN 9058095673, pp. 233238.

Smithe, D., Myra, J.R. \& D'Ippolito, D.A. 2014 Quantitative modeling of icrf antennas with integrated time domain $\mathrm{rf}$ sheath and plasma physics. AIP Conference Proceedings 1580, 89-96.

Smithe, D. N. 2007 Finite-difference time-domain simulation of fusion plasmas at radiofrequency time scales. Phys. Plasmas 14, 056104.

StAngeBy, P.C. 2012 The chodura sheath for angles of a few degrees between the magnetic field and the surface of divertor targets and limiters. Nuc. Fusion 52, 083012.

Stix, T. H. 1992 In Waves in Plasmas. AIP.

Swanson, A. 2003 In Plasma Waves. IOP pusblishing 2nd ed.

Sysoeva, E. V., da Silva, F., Gusakov, E. Z., Heuraux, S. \& Popov, A. Yu 2014 Erch microwave beam broadening in the edge turbulent plasma. AIP Conf. Proc. 1522, 522-525.

Sysoeva, E. V., da Silva, F., Gusakov, E. Z., Heuraux, S. \& Popov, A. Yu 2015 Ecrh beam broadening in the edge turbulent plasma of fusion machines. Nuc. Fusion. 55, 033016.

Taflove, A. \& Hagness, S.C. 2000 In Computational Electrodynamics: the finite-difference time-domain method, pp. 175-235. Artech House.

Tenouxa, T. \& Lostanlen, Y. 2012 Ecrh beam broadening in the edge turbulent plasma of fusion machines. Physical Communication 5, 338351.

Ticos, C.M., Stoica, D.S. \& Delzanno, G.L. 2012 Generation of dust projectiles passing over an obstacle in the plasma sheath. Phys. Plasmas 19, 083701.

Tierens, W. \& Zutter, D. De 2012 Finite-temperature corrections to the time-domain equations of motion for perpendicular propagation in nonuniform magnetized plasmas. Phys. Plasmas 19, 112110.

Tsironis, C., Peteers, A. G., Isliker, H., Strinzi, D. \& Chatziantonaki, I. 2009 Electron cyclotron wave scattering by edge density fluctuations in iter. Phys. Plasmas 16, 112510.

Wilson, J. R. \& Bonoli, P. 2015 Progress on ion cyclotron range of frequencies heating 
physics and technology in support of the international tokamak experimental reactor. Phys. of Plasmas 22, 021801.

Wright, J. C., Valeo, E. J., Phillips, C. K., Bonoli, P. T. \& Brambilla, M. 2008 Full wave simulations of lower hybrid waves in toroidal geometry with non-maxwellian electrons. Comm. Comp. Phys. 4, 545-555.

Wukitch, S. J., LaBombard, B., Lin, Y. \& Lipschultz, B. et al 2009 Icrf specific impurity sources and plasma sheaths in alcator c-mod. J. Nucl. Mater. 390-391, 951954. 